\title{
Atracción, deseos y conductas sexuales: Evidencias de la diversidad en la orientación sexual de jóvenes españoles
}

\section{Sexual attraction, desires and behaviors: Evidence of diversity in sexual orientation among Spanish youth}

Fecha de recepción: 24-07-2020

Fecha de aceptación: 12-11-2020
Juan E. Nebot-García

Salusex,Universitat Jaume I de Castellón. España

Rafael Ballester-Arnal

Salusex, Universitat Jaume I de Castellón. España

Estefanía Ruiz-Palomino

Salusex, Universitat Jaume I de Castellón. España

\section{resumen/alsstract:}

La orientación sexual ha sido una de las dimensiones sexuales que más se ha intentado normativizar, invisibilizando las realidades que se alejaban de la heterosexualidad. Por eso, este estudio pretende profundizar en las experiencias sexuales con el mismo sexo y el opuesto en mujeres y hombres jóvenes. 500 mujeres y 500 hombres españoles, de entre 18 y 25 años, respondieron un cuestionario ad hoc sobre diversidad sexual. La edad media de la muestra fue de 21.23 años ( $D T=1.99$ ). Un $26.1 \%$ de los participantes se identificaba con una orientación diferente a la heterosexual, siendo el $12.8 \%$ bisexuales y el 10.8\% homosexuales. En general, las mujeres refieren sentir mayor atracción sexual por ambos sexos, así como mayores prevalencias de conductas sexuales y mayor disposición a realizarlas con ambos sexos. La sexualidad es más compleja de lo que se estimaba tradicionalmente, observándose gran diversidad de comportamientos, independientemente incluso de la orientación sexual autoadscrita. Estos resultados podrían ser útiles, en terapia, para trabajar la autoaceptación y normalizar la propia diversidad y, como prevención, para educar en la tolerancia y el respeto, reducir el estigma del colectivo LGTBI y flexibilizar en la población general algunas ideas asumidas sobre la sexualidad.

Sexual orientation has been one of the sexual dimensions that has been more standardized, making invisible the realities that were moving away from heterosexuality. For this reason, this study aims to delve into sexual experiences with the same and the opposite sex in young women and men. 500 Spanish women and 500 Spanish men, between 18 and 25 years old, answered an ad hoc questionnaire about sexual diversity. The mean age of the sample was 21.23 years old $(S D=1.99)$. A $26.1 \%$ of the participants identified with a different orientation than heterosexual, a $12.8 \%$ as a bisexual and a $10.8 \%$ as a homosexual. In general, women feel greater sexual attraction for both sexes, as well as a higher prevalence of sexual behavior and a greater willingness to engage in sexual behaviors with both sexes. Sexuality is more complex than traditionally estimated, a wide variety of behaviors is observed regardless of self-assigned sexual orientation. These results could be useful, in therapy, to work on self-acceptance and normalize one's sexual diversity and, as prevention, to educate in tolerance, reduce the stigma of the LGTBI community and make flexible concepts about sexuality.

\section{palabras clave/keywords:}

diversidad sexual, orientación sexual, jóvenes, diferencias de género.

sexual diversity, sexual orientation, youth, gender differences. 


\section{Introducción}

La sexualidad y, más concretamente, la orientación sexual son dos ámbitos de nuestras vidas en los que más represión se ha podido observar, intentando normativizar todas las vivencias posibles en una sola categoría: la heterosexualidad. Esta era la única orientación sexual que se consideraba válida, según lo que dictaba la cultura y las normas sociales (Eaton y Rios, 2017), relegando al resto de vivencias a la anormalidad o, incluso, llegando a ser consideradas como una enfermedad (Carroll, 2015). Sin embargo, la evidencia científica ha demostrado que la sexualidad es una dimensión flexible y diversa, siendo vivida de forma diferente por cada persona (Manley, Diamond y van Anders, 2015).

Algunos de los estudios que sirvieron para romper con esta concepción tradicional de la orientación sexual fueron los de Alfred Kinsey y Fritz Klein. En los años cincuenta, Kinsey realizó dos estudios (Kinsey, Pomeroy y Martin, 1948; Kinsey, Pomeroy, Martin y Gebhard, 1953) en los que se observó que, además de conductas con el sexo opuesto, las personas entrevistadas mostraban altas prevalencias de conductas sexuales con individuos del mismo sexo, independientemente de la orientación sexual autoadscrita. Además, en los estudios de Kinsey se utilizaron unas escalas Likert, que consistían en una gradación de 7 puntos que iban de conductas "exclusivamente heterosexuales" a conductas "exclusivamente homosexuales". Estas escalas sirvieron para dar visibilidad a la bisexualidad y empezar a concebir la orientación sexual como una dimensión con diferentes posiciones intermedias. Años más tarde, en 1978, Fritz Klein (Klein, 2012) observó que los estudios de Kinsey tenían algunas limitaciones y elaboró un modelo mucho más completo, en el que se concibe a la orientación sexual como un constructo formado por diversas dimensiones (como la conducta sexual, la atracción sexual, las fantasías sexuales, etc.) que fluctúan en función del tiempo, es decir, la orientación sexual sería multidimensional y flexible.

Pese a que todos estos estudios sirvieron para flexibilizar los conceptos tradicionales y categoriales sobre la orientación sexual, estas etiquetas sexuales siguen estando muy presentes en la sociedad, siendo utilizadas por la gran mayoría de la población para incluirse dentro de un grupo de personas con los mismos intereses, conductas y deseos sexuales. Además, este sentimiento de pertenencia y de identificación es parte del proceso por el que pasa el ser humano para culminar la toma de conciencia de su orientación sexual (Dillon, Worthington y Moradi, 2011).

No obstante, estas categorías sexuales (heterosexual, homosexual, bisexual), lejos de ser perfectas y absolutas, albergan a personas con diferentes vivencias sexuales. La evidencia científica muestra que, personas con una misma orientación sexual autoadscrita, pueden presentar diferentes comportamientos, deseos y atracciones sexuales. Tal es así que, estudios recientes muestran que personas que se sitúan dentro de una categoría de orientación monosexual, como la heterosexualidad o la homosexualidad, pueden mostrar atracción, deseos o conductas sexuales por el sexo no preferente (Ballester-Arnal y Gil-Llario, 2016, 2019; Copen, Chandra y Febo-Vazquez, 2016; Nebot-Garcia, García-Barba, Gil-Juliá, GiménezGarcía y Ballester-Arnal, 2018; Vrangalova y Savin-Williams, 2012). Suele considerarse socialmente que la etiqueta con la que una persona se identifica es el único criterio que, de 
forma completa y absoluta, define la orientación sexual. Sin embargo, existen diferentes áreas y dimensiones a tener en cuenta a la hora de evaluar hacia quién se dirige la atracción y los deseos sexuales. Cabría realizar una gradación de conductas sexuales que explorara experiencias más básicas, como considerar a alguien guapo/a o atractivo/a, pasando por fantasías sexuales, sueños eróticos, deseos sexuales, y que terminara evaluando experiencias más explícitas, como mantener relaciones sexuales. Todo ello, explorándolo con personas del mismo sexo y del sexo opuesto. De esta manera, se podría explorar si la incongruencia que se observaba en estudios pasados entre la orientación sexual autoadscrita y las diferentes experiencias sexuales (Ballester-Arnal y Gil-Llario, 2016, 2019; Copen et al., 2016; Nebot-Garcia et al., 2018; Vrangalova y Savin-Williams, 2012) se observa también en todas las conductas del continuo, independientemente del carácter más básico o explícito de las experiencias.

Esta disonancia entre lo que las personas experimentan y lo que se espera de ellas (incluso lo que ellas mismas esperan de sí mismas) debido a la orientación sexual con la que se identifican, puede generar malestar psicológico, sobre todo si estas vivencias discordantes van acompañadas de dudas con respecto a la propia orientación sexual. Todo ello puede afectar al bienestar psicológico y desencadenar algún problema de salud mental, como pueden ser síntomas depresivos o ansiedad (Borgogna, McDermott, Aita y Kridel, 2019; Shearer et al., 2016).

La influencia normativa parece repercutir de manera diferencial en hombres y mujeres. A los hombres se les permite menos explorar su orientación sexual, debiendo ajustarse más a los roles de género tradicionales y a comportamientos marcadamente masculinos (Cornejo, 2015), castigándose cualquier conducta que pudiera ser considerada homosexual (Poteat y Anderson, 2012) o femenina (Carnaghi, Maass y Fasoli, 2011). En cambio, esta exploración sexual está más aceptada en las mujeres, que incluso suelen ser incitadas y animadas a que tengan ciertos comportamientos homosexuales, ya que las conductas lésbicas son consideradas por los hombres heterosexuales como una fantasía sexual (Yost y McCarthy, 2012).

Pese a lo importante que es tener una vivencia plena y libre de la sexualidad para tener una buena calidad de vida (Greenberg, Smith, Kim, Naghdechi y IsHak, 2017), sobre todo en población joven, que es cuando empieza a consolidarse la identidad y a tomarse conciencia de la orientación sexual (Soriano, 2004), en España existe poca literatura que explore con profundidad la diversidad sexual. Con la intención de subsanar esta necesidad, el objetivo de este trabajo ha sido profundizar en diferentes dimensiones de la sexualidad para tratar de visibilizar la diversidad de atracciones, deseos y conductas sexuales existentes en la sociedad.

\section{Método}

\section{Participantes}

A partir de un muestreo aleatorio simple en la población general, se recogieron un total de 4564 respuestas. Para formar parte del presente estudio, los participantes debían cumplir con los criterios de inclusión: tener entre 18 y 25 años, identificarse como hombre o mu- 
jer, y residir en España. A partir de estos criterios, se seleccionaron a 1000 personas, 500 hombres y 500 mujeres, de entre 18 y 25 años de edad $(M=21.23$; DT=1.99). Los chicos tenían una media de edad de 21.30 años $(\mathrm{DT}=2.01)$ y las chicas de 21.15 años $(\mathrm{DT}=1.97)$ $(\mathrm{d}=.074 ; \mathrm{LI}=-.049 ; \mathrm{LS}=.198)$. La mayoría indicaron ser ateos o agnósticos $(69.4 \%)$, con una ideología política progresista $(69 \%)$, con estudios universitarios $(69 \%)$ y procedían de una localidad urbana (68.6\%). Para ver información más detallada y separada por género, véase la Tabla 1.

Tabla 1.- Principales características sociodemográficas de la muestra.

\begin{tabular}{|c|c|c|c|c|}
\hline & & Hombre & Mujer & \multirow[t]{2}{*}{$\begin{array}{l}\text { Tamaño } \\
\text { del efecto }\end{array}$} \\
\hline & & $\%$ & $\%$ & \\
\hline \multirow[t]{3}{*}{ Creencias religiosas } & Creyente practicante & 5.4 & 5.6 & \multirow{3}{*}{$\mathrm{V}=.045$} \\
\hline & Creyente no practicante & 23.2 & 27 & \\
\hline & Ateo o agnóstico & 71.4 & 67.4 & \\
\hline \multirow[t]{7}{*}{ Nivel de estudios } & Sin estudios & 0.2 & 0.2 & \multirow{7}{*}{$\mathrm{V}=.107$} \\
\hline & Primarios & 1 & 0.6 & \\
\hline & Secundarios & 21.6 & 16.2 & \\
\hline & Formación profesional & 10.8 & 11.4 & \\
\hline & Diplomatura & 2 & 0.4 & \\
\hline & Licenciatura/Grado & 58.6 & 64.8 & \\
\hline & Máster/Doctorado & 5.8 & 6.4 & \\
\hline \multirow{4}{*}{$\begin{array}{l}\text { Lugar de residencia } \\
\text { durante infancia/ } \\
\text { adolescencia }\end{array}$} & Urbana de costa & 35.6 & 29.4 & \multirow{4}{*}{$\mathrm{V}=.067$} \\
\hline & Urbana de interior & 34.8 & 37.4 & \\
\hline & Rural de costa & 6 & 7.2 & \\
\hline & Rural de interior & 23.6 & 26 & \\
\hline \multirow[t]{4}{*}{ Ideología política } & Conservador & 8.8 & 3.6 & \multirow{4}{*}{$V=.132$} \\
\hline & Centro & 51 & 49.6 & \\
\hline & Progresista & 21.2 & 20.2 & \\
\hline & Indiferente & 19 & 26.6 & \\
\hline
\end{tabular}

\section{Instrumentos}

Para este estudio se utilizó un cuestionario de 94 ítems, desarrollado ad hoc por el grupo de investigación Salusex de las Universidades Jaume I de Castellón y de Valencia en el año 2017, en el que se pretendía evaluar la amplitud y diversidad de la vivencia de la orientación sexual en diferentes ámbitos. Para este trabajo se analizaron un total de 29 ítems, agrupados en los siguientes cuatro ámbitos: 
Orientación sexual. En esta escala se pregunta la orientación sexual con la que se identifican, debiendo elegir entre "heterosexual", "homosexual", "bisexual", "asexual" u "otro". En caso de seleccionar la opción de "otro", se les pedía que especificaran con qué etiqueta se identificaban mejor.

Atracción sexual. En este ítem, adaptado de Kinsey (1948), se pregunta “¿cuál de las siguientes afirmaciones describe mejor hacia quién te sientes atraído/a sexualmente?". Los participantes pueden contestar siguiendo una escala tipo Likert que oscila desde 1 ("Me siento atraído/a solo hacia el otro sexo") a 7 ("Me siento atraído/a solo hacia mi mismo sexo"). Existe otra opción, además, reservada para aquellas personas asexuales: "No me siento atraído/a por ningún sexo".

Gradación de experiencias estético-eróticas. Este apartado consta de 12 ítems mediante los que se mide si existe algún tipo de apreciación de la belleza estética o de experiencia erótica. Estas conductas están separadas en dos escalas de 6 ítems cada una: una aborda las experiencias con personas del mismo sexo y la otra, con personas del sexo opuesto. En todas ellas debe contestarse "sî" o "no" a preguntas como si alguna vez han considerado que alguien era guapo/a (ítem 1), o atractivo/a (ítem 2), si han tenido sueños eróticos (ítem 3), fantasías (ítem 4) o deseo de tener relaciones sexuales (incluyendo tocamientos y/o masturbación) con alguien (ítem 5), o si, finalmente, han tenido relaciones sexuales (incluyendo tocamientos y/o masturbación) (ítem 6). La escala sobre el mismo sexo presenta una fiabilidad evaluada a través de la consistencia interna de .792 y la del sexo opuesto, de .748 .

Intención de conducta. En este apartado se pregunta la disposición a realizar dieciséis conductas diferentes, separadas en dos escalas: ocho con el mismo sexo y ocho con el sexo opuesto.

En cuanto a las preguntas sobre el mismo sexo, seis se presentan a toda la población (besos en la boca, caricias y abrazos desnudos/as, masturbar a la otra persona, recibir masturbación, realizar sexo oral a otra persona, y recibir sexo oral), y dos, solo a los hombres (penetrar analmente, y ser penetrado analmente). Esta escala tiene una consistencia interna de .969 .

Por otra parte, en las preguntas sobre el sexo opuesto, se incluyen las conductas anteriores que eran comunes a toda la población (besos en la boca, caricias y abrazos desnudos, masturbar a la otra persona, recibir masturbación, realizar sexo oral a otra persona, y recibir sexo oral), y se añaden dos más (coito vaginal y coito anal). Esta escala presenta una consistencia interna de .949 .

Todas estas conductas deben ser respondidas atendiendo a las siguientes opciones de respuesta: "De ninguna manera", "Solo si la otra persona es atractiva", "Solo si es de confianza" o "Fácilmente".

\section{Procedimiento}

Para este estudio se siguió un diseño transversal, en el que se tomaron los datos de todas las variables en el mismo momento temporal. Durante los meses de enero a marzo del 2017, se hizo difusión de un anuncio que animaba a participar en un estudio sobre orientación se- 
xual. Con el objetivo de llegar a toda la geografía española, se difundió el estudio por redes sociales y foros de interés de diferentes provincias españolas. Cuando los participantes seleccionaban el anuncio, antes de poder empezar a contestar el cuestionario on-line, accedían a una pantalla en la que se les informaba del carácter anónimo, voluntario y confidencial de la investigación, y se les pedía el consentimiento informado. La investigación contaba con el permiso de la Comisión Deontológica de la Universitat Jaume I (Castellón, España) y, en todo momento, se siguieron los principios éticos de la Declaración de Helsinki.

\section{Análisis estadísticos}

Se realizaron distintos análisis mediante el programa estadístico SPSS versión 25. En primer lugar, se realizaron descriptivos para conocer las prevalencias de las diferentes variables y, para conocer si existían diferencias entre hombres y mujeres, se utilizaron pruebas de Chi cuadrado. Para facilitar el análisis y la interpretación de los resultados, se agruparon las respuestas intermedias en la escala de Intención de Conducta ("Solo si es atractivo/a" y "Solo si es de confianza"), recodificándose como "Depende".

\section{Resultados}

\section{Orientación sexual autoadscrita: diferencias de género}

En cuanto a la orientación sexual, el 73.9\% de los participantes se identificaban como heterosexuales, el $10.8 \%$ como homosexuales, el $12.8 \%$ como bisexuales, y el $2.7 \%$ restante, se identificaban con otras orientaciones.

Tal y como se observa en la Figura 1, hombres y mujeres se identifican, en mayor medida, con la heterosexualidad. No obstante, existen diferencias entre hombres y mujeres en la segunda opción más prevalente: los hombres se sitúan más en la homosexualidad y las mujeres, en la bisexualidad. Estas diferencias en las prevalencias fueron estadísticamente significativas en función del género $\left(\chi^{2}=54.69 ; \mathrm{p}<.001\right)$.

\section{Atracción sexual manifestada: diferencias de género}

Por lo que respecta a la atracción sexual medida a través de la Escala Kinsey (véase Figura 2), el $52.4 \%$ de las mujeres y el $61.6 \%$ de los hombres solo siente atracción hacia el otro sexo. Por otra parte, un $2.4 \%$ de mujeres y un $11.8 \%$ de hombres siente atracción solo hacia el mismo sexo. El $0.8 \%$ de hombres y el $0.8 \%$ de mujeres no sienten atracción sexual por nadie. Finalmente se observa que el $25.8 \%$ de hombres y el $44.4 \%$ de mujeres muestran algún grado de atracción por ambos sexos. Todas estas diferencias encontradas en la atracción sexual de hombres y mujeres fueron estadísticamente significativas $\left(\chi^{2}=81.80 ; p<.001\right)$.

\section{Gradación de experiencias estético-eróticas hacia el otro y el mismo sexo: diferencias de género}

Los resultados sobre las diferentes experiencias estético-eróticas con personas del otro sexo muestran mayores prevalencias en las mujeres que en los hombres (véase Tabla 2), pero solo se observaron diferencias estadísticamente significativas en función del género en las 
Figura 1.- Diferencias de género en la orientación sexual autoadscrita.

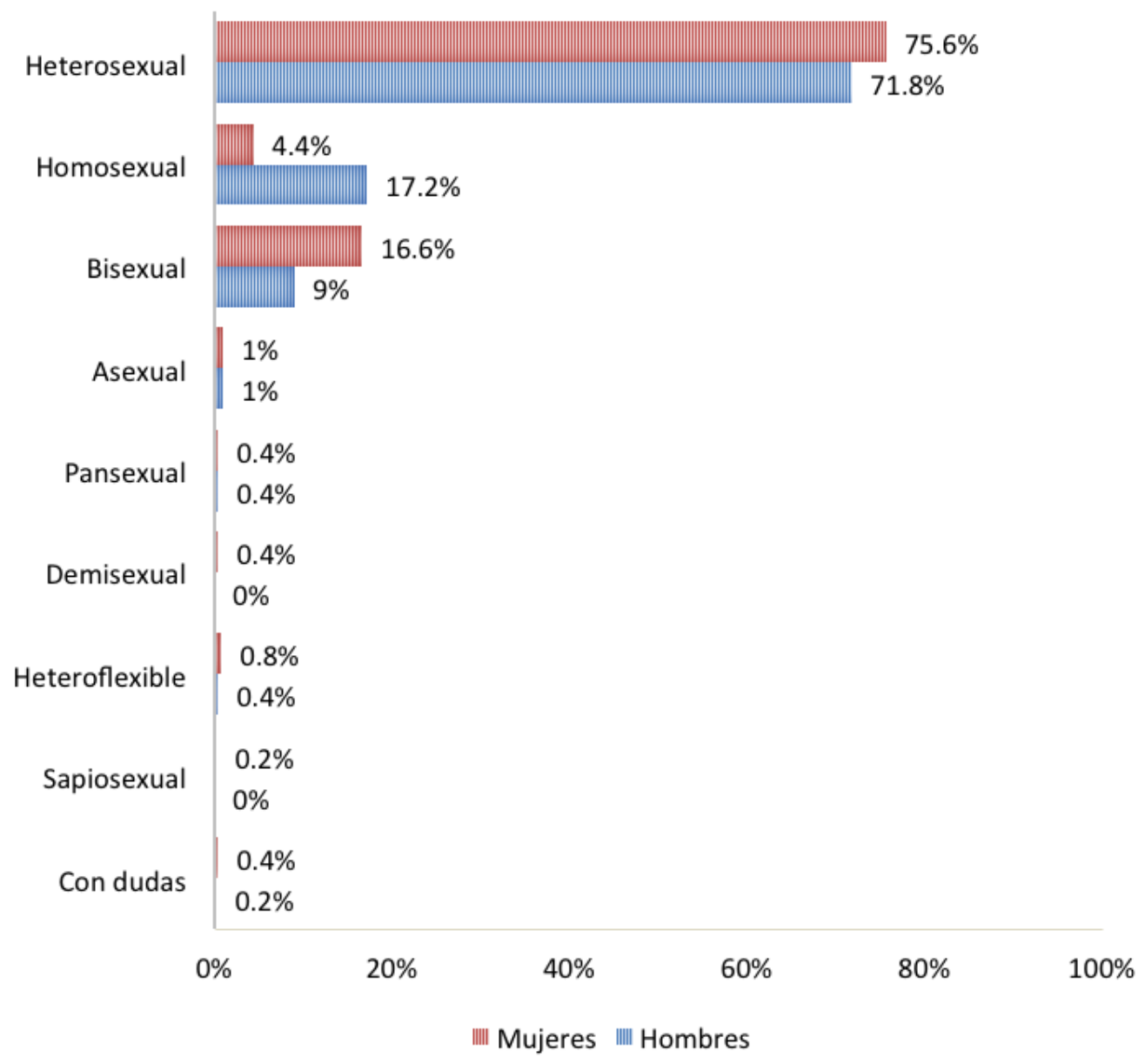

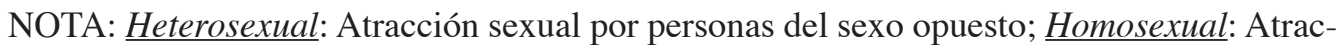
ción sexual por personas del mismo sexo; Bisexual: Atracción sexual por hombres y mujeres, aunque también hay gente bisexual que siente atracción por hombres, mujeres y personas no binarias (personas que no se identifican como hombres o mujeres); Asexual: No sienten atracción sexual hacia ninguna persona; Pansexual: Atracción sexual por personas de cualquier sexo o género; Demisexual: Solo siente atracción sexual por personas con las que tiene un vínculo emocional muy fuerte; Heteroflexible: Atracción sexual por personas del sexo opuesto aunque, en algunas ocasiones, puede sentir atracción o tener relaciones sexuales con personas del mismo sexo; Sapiosexual: Atracción sexual hacia personas con gran inteligencia. 
Figura 2.- Diferencias de género en la atracción sexual según Kinsey.

Me siento atraído/a solo hacia el otro sexo

Me siento atraído/a casi siempre hacia el otro sexo y en muy contadas ocasiones hacia mi mismo sexo

Me siento atraído/a algo más hacia el otro sexo que hacia mi mismo sexo

Me siento igualmente atraído/a hacia cualquier sexo

Me siento atraído/a algo más hacia mi mismo sexo que hacia el otro sexo

Me siento atraído/a casi siempre hacia mi mismo sexo y en muy contadas ocasiones hacia el otro sexo

Me siento atraído/a solo hacia mi mismo
sexo No me siento atraído/a por ningún sexo

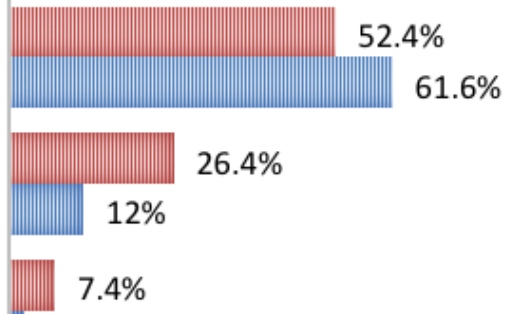

$2.4 \%$

$3.8 \%$

$1.6 \%$

$2.2 \%$

$3 \%$

\section{$4.6 \%$}

$6.8 \%$

$2.4 \%$

$11.8 \%$

$0.8 \%$

$0.8 \%$

\section{$0 \% \quad 20 \% \quad 40 \% \quad 60 \% \quad 80 \% \quad 100 \%$ \\ IIIII Mujeres ${ }^{\text {IIIII Hombres }}$}

relaciones sexuales, los deseos sexuales y en el hecho de ver atractivo/a a alguien del otro sexo.

Tanto para hombres como para mujeres, la conducta más prevalente es la de haber considerado guapa a una persona del otro sexo. No obstante, hombres y mujeres difieren en la experiencia menos común. Así pues, la conducta con el sexo opuesto menos experimentada por los hombres es la de mantener relaciones sexuales, mientras que en las mujeres es la de tener fantasías sexuales.

En cuanto a las experiencias estético-eróticas con personas del mismo sexo (véase Tabla 2), las mujeres muestran mayores prevalencias en casi todas las variables, a excepción de las relaciones sexuales, que es más común en los hombres. Estas diferencias fueron estadísticamente significativas en función del género en todas las variables menos en las fantasías sexuales. 
Hombres y mujeres refieren que la experiencia más común con el mismo sexo es la de haber considerado a alguien guapo/a, y la menos prevalente, la de haber mantenido relaciones sexuales.

Tabla 2.- Análisis diferenciales, según el género, en las experiencias estético-eróticas.

\begin{tabular}{lccc}
\hline & $\begin{array}{c}\text { Hombres } \\
\mathbf{\%}\end{array}$ & $\begin{array}{c}\text { Mujeres } \\
\mathbf{\%}\end{array}$ & $\chi^{\mathbf{2}}(\mathbf{p})$ \\
\hline Otro sexo & 75.6 & 93.4 & $60.47(<.001)$ \\
Relaciones sexuales & 86 & 92.8 & $29.02(<.001)$ \\
Deseos sexuales & 83.2 & 86.2 & $1.73(.188)$ \\
Fantasías sexuales & 88.6 & 92.2 & $3.77(.053)$ \\
Sueños eróticos & 95.2 & 98.2 & $7.05(.008)$ \\
Atractivo/a & 99.4 & 99.6 & $0.20(.654)$ \\
Guapo/a & & & \\
Mismo sexo & 24.6 & 17.8 & $6.92(.009)$ \\
Relaciones sexuales & 36.2 & 46.4 & $10.72(.001)$ \\
Deseos sexuales & 32.4 & 31.4 & $0.11(.734)$ \\
Fantasías sexuales & 35.8 & 50.8 & $22.91(<.001)$ \\
Sueños eróticos & 79.6 & 95.2 & $55.24(<.001)$ \\
Atractivo/a & 89.2 & 97.6 & $28.61(<.001)$ \\
Guapo/a & & &
\end{tabular}

\section{Intención de conductas eróticas hacia el otro y el mismo sexo: diferencias de género}

En cuanto a la intención de realizar ciertas conductas sexuales con alguien del otro sexo (véase Tabla 3), la conducta de "besos en los labios" es la que mayor disposición muestra a ser realizada por hombres y mujeres. Por su parte, practicar coito anal con alguien del otro sexo es la conducta que menos disposición muestra en mujeres y hombres, pero al mismo tiempo, también es la variable en la que se obtienen mayores diferencias entre ambos.

Por lo que respecta a las diferencias de género, las mujeres muestran mayor disposición en casi todas las variables, a excepción del coito anal, en la que los hombres muestran mayor disposición a realizarla. Todas estas diferencias fueron estadísticamente significativas entre hombres y mujeres, menos en la conducta de "besos en los labios". 
Tabla 3.- Diferencias de género en la intención de conductas eróticas con personas del otro sexo.

\begin{tabular}{|c|c|c|c|c|}
\hline & & $\begin{array}{c}\text { Hombre } \\
\% \\
\end{array}$ & $\begin{array}{c}\text { Mujer } \\
\%\end{array}$ & $\chi^{2}(\mathbf{p})$ \\
\hline \multirow[t]{3}{*}{ Besos en los labios } & De ninguna manera & 3.4 & 2.6 & \multirow{3}{*}{$\begin{array}{c}3.58 \\
(.166)\end{array}$} \\
\hline & Depende & 34.8 & 40.4 & \\
\hline & Fácilmente & 61.8 & 57 & \\
\hline \multirow[t]{3}{*}{ Abrazos y caricias desnudos } & De ninguna manera & 10.8 & 5.6 & \multirow{3}{*}{$\begin{array}{c}42.13 \\
(<.001)\end{array}$} \\
\hline & Depende & 39.6 & 59.8 & \\
\hline & Fácilmente & 59.8 & 34.6 & \\
\hline \multirow[t]{3}{*}{ Masturbar a la otra persona } & De ninguna manera & 14.4 & 7.2 & \multirow{3}{*}{$\begin{array}{c}51.75 \\
(<.001)\end{array}$} \\
\hline & Depende & 39.4 & 61.8 & \\
\hline & Fácilmente & 46.2 & 31 & \\
\hline \multirow[t]{3}{*}{ Recibir masturbación } & De ninguna manera & 14 & 6.2 & \multirow{3}{*}{$\begin{array}{c}71.76 \\
(<.001)\end{array}$} \\
\hline & Depende & 35.8 & 62.2 & \\
\hline & Fácilmente & 50.2 & 31.6 & \\
\hline \multirow[t]{3}{*}{ Realizar sexo oral } & De ninguna manera & 16.2 & 10.8 & \multirow{3}{*}{$\begin{array}{c}47.83 \\
(<.001)\end{array}$} \\
\hline & Depende & 41.8 & 63.6 & \\
\hline & Fácilmente & 42 & 25.6 & \\
\hline \multirow[t]{3}{*}{ Recibir sexo oral } & De ninguna manera & 14.2 & 8 & \multirow{3}{*}{$\begin{array}{c}73.99 \\
(<.001)\end{array}$} \\
\hline & Depende & 36.2 & 63.4 & \\
\hline & Fácilmente & 49.6 & 28.6 & \\
\hline \multirow[t]{3}{*}{ Coito vaginal } & De ninguna manera & 14.6 & 6.6 & \multirow{3}{*}{$\begin{array}{c}66.46 \\
(<.001)\end{array}$} \\
\hline & Depende & 38 & 63.4 & \\
\hline & Fácilmente & 47.4 & 30 & \\
\hline \multirow[t]{3}{*}{ Coito anal } & De ninguna manera & 24.8 & 52 & \multirow{3}{*}{$\begin{array}{l}128.13 \\
(<.001)\end{array}$} \\
\hline & Depende & 40.2 & 39.4 & \\
\hline & Fácilmente & 35 & 8.6 & \\
\hline
\end{tabular}


Tabla 4.- Diferencias de género en la intención de conductas eróticas con personas del mismo sexo.

\begin{tabular}{|c|c|c|c|c|}
\hline & & $\begin{array}{c}\text { Hombre } \\
\%\end{array}$ & $\begin{array}{c}\text { Mujer } \\
\%\end{array}$ & $\chi^{2}(\mathbf{p})$ \\
\hline \multirow[t]{3}{*}{ Besos en los labios } & De ninguna manera & 41.4 & 13.2 & \multirow{3}{*}{$\begin{array}{l}100.33 \\
(<.001)\end{array}$} \\
\hline & Depende & 36 & 52 & \\
\hline & Fácilmente & 22.6 & 34.8 & \\
\hline \multirow[t]{3}{*}{ Abrazos y caricias desnudos/as } & De ninguna manera & 60.4 & 41.2 & \multirow{3}{*}{$\begin{array}{c}41.73 \\
(<.001)\end{array}$} \\
\hline & Depende & 24.6 & 42.2 & \\
\hline & Fácilmente & 15 & 16.6 & \\
\hline \multirow[t]{3}{*}{ Masturbar a la otra persona } & De ninguna manera & 63.4 & 52.8 & \multirow{3}{*}{$\begin{array}{c}18.88 \\
(<.001)\end{array}$} \\
\hline & Depende & 22.4 & 34.8 & \\
\hline & Fácilmente & 14.2 & 12.4 & \\
\hline \multirow[t]{3}{*}{ Recibir masturbación } & De ninguna manera & 61 & 47.6 & \multirow{3}{*}{$\begin{array}{c}27.67 \\
(<.001)\end{array}$} \\
\hline & Depende & 23 & 38.2 & \\
\hline & Fácilmente & 16 & 14.2 & \\
\hline \multirow[t]{3}{*}{ Realizar sexo oral } & De ninguna manera & 65.4 & 58.4 & \multirow{3}{*}{$\begin{array}{l}13.33 \\
(.001)\end{array}$} \\
\hline & Depende & 21.6 & 31.6 & \\
\hline & Fácilmente & 13 & 10 & \\
\hline \multirow[t]{3}{*}{ Recibir sexo oral } & De ninguna manera & 61.4 & 50.4 & \multirow{3}{*}{$\begin{array}{c}19.72 \\
(<.001)\end{array}$} \\
\hline & Depende & 23.4 & 36.2 & \\
\hline & Fácilmente & 15.2 & 13.4 & \\
\hline \multirow[t]{3}{*}{ Penetrar analmente* } & De ninguna manera & 66.8 & - & \multirow{3}{*}{-} \\
\hline & Depende & 20.8 & - & \\
\hline & Fácilmente & 12.4 & - & \\
\hline \multirow[t]{3}{*}{ Ser penetrado analmente* } & De ninguna manera & 69.6 & - & \multirow{3}{*}{-} \\
\hline & Depende & 19.8 & - & \\
\hline & Fácilmente & 10.6 & - & \\
\hline
\end{tabular}

*Estas variables solo fueron evaluadas en los hombres. 
Por lo que respecta a la disposición a realizar ciertas conductas sexuales con alguien del mismo sexo (véase Tabla 4), la conducta de "besos en los labios" es la que mayor disposición muestra a ser realizada, pero también es la que muestra mayores diferencias entre mujeres y hombres. Por su parte, la opción de "realizar sexo oral a otra persona" y la de "ser penetrado analmente" son las que menos disposición muestran en mujeres y hombres, respectivamente. En todas las variables estudiadas se observan diferencias significativas entre hombres y mujeres, siendo las mujeres quienes presentan mayor disposición a realizarlas.

\section{Discusión}

A la luz de los resultados se puede confirmar que la sexualidad es mucho más compleja y diversa de lo que se ha estimado tradicionalmente. Estos hallazgos evidencian que en las etiquetas sexuales tradicionales relacionadas con la orientación sexual se agrupan personas que presentan comportamientos, atracciones y deseos muy diferentes, quedando patente la flexibilidad de las categorías propuestas tradicionalmente como estancas, y siendo las mujeres las que presentan mayor variabilidad de respuestas.

Por lo que respecta a los resultados obtenidos, se observa que no existe concordancia entre los porcentajes de las orientaciones autoadscritas y los de la atracción sexual manifestada medidos a través de la Escala Kinsey, tal y como ya se había sugerido en otros estudios (Ballester-Arnal y Gil-Llario, 2016, 2019; Copen et al., 2016; Nebot-Garcia et al., 2018; Vrangalova y Savin-Williams, 2012). En cuanto a las prevalencias, la orientación sexual más común es la heterosexual, tanto para hombres como para mujeres. No obstante, como segunda orientación en prevalencia aparece la homosexualidad en los hombres y la bisexualidad en las mujeres (Gates, 2011; Petersen y Hyde, 2011; Vrangalova y Savin-Williams, 2012). Estos niveles de atracción bisexual se incrementan cuando se evalúa en función de la gradación de Kinsey, siendo las mujeres quienes presentan mayor atracción por ambos sexos. En cambio, los hombres, tienden a situarse más en los extremos, identificándose más con la monosexualidad (Ballester-Arnal y Gil-Llario, 2016; Copen et al., 2016).

En términos generales, en cuanto a la gradación de experiencias estético-eróticas y a la intención de conducta, tanto con el mismo sexo como con el sexo opuesto, las mujeres son las que muestran mayores prevalencias. Estos hallazgos concuerdan con los resultados explicados en el párrafo anterior, en el que se sostenía que las mujeres presentaban mayor interés sexual por ambos sexos. Una posible explicación a estas mayores prevalencias en mujeres podría deberse a la fluidez sexual que se ha observado en las mujeres (Diamond, 2008), que, independientemente de su orientación sexual, podrían llegar a experimentar atracción por gente del sexo no preferente. Además, las mujeres se cuestionan más su sexualidad (Morgan, Steiner y Thompson, 2010; Morgan y Thompson, 2011), pudiendo ser esa una de las causas por las que realizan más conductas sexuales con hombres y mujeres. Por otra parte, la rigidez de las etiquetas sexuales (Davis-Delano y Morgan, 2016; Davis-Delano, Morgan, Gillard y Davis, 2018) es mayor en los hombres. Además, los hombres presentan mayores niveles de homofobia, sobre todo hacia los hombres gays (Petersen y Hyde, 2011); y la presencia de conductas homosexuales está más castigada en ellos (Herek, 2009), lo que podría explicar una menor exploración sexual. 
Por otra parte, se observa que las experiencias más básicas, como pueden ser considerar a alguien guapo/a o darse besos en los labios, son las variables en las que se obtienen mayores prevalencias. No obstante, a medida que el contenido sexual de estas variables va haciéndose más manifiesto, como es el caso de mantener relaciones sexuales, el porcentaje de hombres y de mujeres que ha realizado o realizaría este tipo de conductas, disminuye. Evidentemente, el hecho de haber experimentado este tipo de conductas, ya sea con alguien del mismo sexo o del sexo opuesto, no define la orientación sexual de una persona. Es decir, considerar que una persona del mismo sexo es guapa, o haber tenido fantasías o relaciones con alguien del mismo sexo, no significa necesariamente que se sea homosexual. Pero este tipo de experiencias podrían generar un conflicto interno o gran malestar a personas con homofobia o prejuicios sobre los límites de las distintas orientaciones sexuales.

Si bien este estudio ofrece información muy valiosa, no está exento de algunas limitaciones. Una de ellas tiene que ver con la deseabilidad social que podemos encontrarnos en los autoinformes, sobre todo en temas que pueden ir en contra de los propios valores morales o de las normas sociales, como es el caso de la sexualidad y más concretamente de la orientación sexual (Sutton, 2016). No obstante, el hecho de que la recopilación de respuestas haya sido completamente on-line, ofrece un contexto seguro y confiable para que las personas contesten sinceramente, más que si el entrevistador hubiera estado presencialmente delante. Por otra parte, hay que tener en cuenta las características de la muestra, ya que eran personas jóvenes y, la gran mayoría, con estudios universitarios. Esto podría haber sesgado los resultados, aunque por los datos que se desprenden del último Informe sobre el Sistema Estatal de Indicadores de Educación (Ministerio de Educación y Formación Profesional, 2019), gran parte de la población joven en España tiene estudios universitarios o los está cursando.

Para futuras investigaciones, sería interesante ampliar la muestra a todas las edades para que los resultados se puedan generalizar a toda la población. Del mismo modo, también sería conveniente analizar estas variables en función de la orientación sexual autoadscrita. Por otra parte, se podrían investigar qué factores influyen en la vivencia de la diversidad sexual, cuáles pueden facilitar una vivencia positiva y cuáles pueden generar un sentimiento de malestar, como las actitudes homofóbicas.

En cualquier caso, los resultados obtenidos en este estudio deberían ser tenidos en cuenta a la hora de realizar programas terapéuticos y de educación sexual. Por una parte, en cuanto a la intervención clínica, serviría para trabajar la autoaceptación y normalizar la diversidad de vivencias que puede experimentar uno mismo; y por otra parte, con respecto a los programas de educación sexual, estos resultados serían útiles para trabajar el estigma del colectivo LGTBI (lesbianas, gays, transexuales, bisexuales e intersexuales), educar en la tolerancia y respeto y flexibilizar conceptos, dando a conocer lo diversa que puede ser la sociedad y la sexualidad. 


\section{Referencias bibliográficas}

Ballester-Arnal, R. y Gil-Llario, M. D. (2016). Orientación del deseo sexual: Crisis y decadencia de los modelos categoriales. En A. López de la Llave (Coord.), Sexología positiva: (pp. 59-66). Madrid: Universidad Nacional de Educación a Distancia.

Ballester-Arnal, R. y Gil-Llario, M. D. (2019). Orientación sexual, prácticas, frecuencia, fantasías y uso de pornografía. En M. D. Cil-Llario (Coord.), Sexualidad y calidad de vida en las personas mayores: análisis de la población de Castellón (pp. 39-58). Castellón de la Plana: Dávalos Fletcher.

Borgogna, N. C., McDermott, R. C., Aita, S. L. y Kridel, M. M. (2019). Anxiety and depression across gender and sexual minorities: Implications for transgender, gender nonconforming, pansexual, demisexual, asexual, queer, and questioning individuals. Psychology of Sexual Orientation and Gender Diversity, 6(1), 54-63. https://psycnet.apa.org/doi/10.1037/sgd0000306

Carnaghi, A., Maass, A. y Fasoli, F. (2011). Enhancing masculinity by slandering homosexuals: The role of homophobic epithets in heterosexual gender identity. Personality and Social Psychology Bulletin, 37(12), 16551665. https://doi.org/10.1177/0146167211424167

Carroll, J. L. (2015). Sexuality now: Embracing diversity (5th edition). EEUU: Cengage Learning.

Copen, C. E., Chandra, A. y Febo-Vazquez, I. (2016). Sexual behavior, sexual attraction, and sexual orientation among adults aged 18-44 in the United States: Data from the 2011-2013 National Survey of Family Growth. National Health Statistics Reports, (88), 1-14.

Cornejo, J. (2015). Componentes ideológicos de la homofobia. Límite. Revista de Filosofía y Psicología, 7(26), 85106.

Davis-Delano, L. R. y Morgan, E. M. (2016). Heterosexual identity management: How social context affects heterosexual marking practices. Identity, 16(4), 299-318. https://doi.org/10.1080/15283488.2016.1229611

Davis-Delano, L. R., Morgan, E. M., Gillard, A. y Davis, C. V. (2018). When Heterosexuality is Questioned: Stifling Suspicion Through Public Displays of Heterosexual Identity. Journal of Homosexuality, 65(13), 1683-1708. https://doi.org/10.1080/00918369.2017.1387465

Diamond, L. M. (2008). Sexual fluidity: Understanding women's love and desire. Cambridge, MA: Harvard University Press.

Dillon, F. R., Worthington, R. L. y Moradi, B. (2011). Sexual identity as a universal process. In S. J. Schwartz, K. Luyckx, \& V. L. Vignoles (Eds.), Handbook of identity theory and research (Vols 1 and 2., pp. 649-670). New York, NY: Springer. https://doi.org/10.1007/978-1-4419-7988-9_27

Eaton, A. A. y Rios, D. (2017). Social challenges faced by queer Latino college men: Navigating negative responses to coming out in a double minority sample of emerging adults. Cultural Diversity and Ethnic Minority Psychology, 23(4), 457-467. https://doi.org/10.1037/cdp0000134

Gates, G. (2011). How many people are lesbian, gay, bisexual, and transgender? http://williamsinstitute.law. ucla.edu/wp-content/uploads/Gates-How-Many-People-LGBTApr-2011.pdf

Greenberg J. M., Smith K. P., Kim T. Y., Naghdechi L. y IsHak W. W. (2017) Sex and Quality of Life. In IsHak W. (Eds.), The Textbook of Clinical Sexual Medicine. Springer, Cham

Herek, G. M. (2009). Hate crimes and stigma-related experiences among sexual minority adults in the United States: Prevalence estimates from a national probability sample. Journal of Interpersonal Violence, 24(1), 54-74. https://doi.org/10.1177/0886260508316477 
Kinsey, A., Pomeroy, W. y Martin, C. (1948). Sexual behavior in the human male. Philadelphia: Saunders Company.

Kinsey, A., Pomeroy, W., Martin, C. y Gebhard, P. H. (1953). Sexual behavior in the human female. Indiana University Press.

Klein, F. (2012). La opción bisexual: Segunda edición. California, USA: American Institute of Bisexuality.

Manley, M. H., Diamond, L. M. y van Anders, S. M. (2015). Polyamory, monoamory, and sexual fluidity: A longitudinal study of identity and sexual trajectories. Psychology of Sexual Orientation and Gender Diversity, 2(2), 168-180. https://doi.org/10.1037/sgd0000098

Ministerio de Educación y Formación Profesional (2019). Las cifras de la educación en España. Curso 2016-2017 (edición 2019). Madrid, España: Secretaría General Técnica. Centro de Publicaciones. https://sede. educacion.gob.es/publiventa/las-cifras-dela-educacion-en-espana-estadisticas-e-indicadores-estadistica-2019/ensenanza-espana-estadisticas/22812

Morgan, E. M. y Thompson, E. M. (2011). Processes of sexual orientation questioning among heterosexual women. Journal of Sex Research, 48(1), 16-28. https://doi.org/10.1080/00224490903370594

Morgan, E. M., Steiner, M. G. y Thompson, E. M. (2010). Processes of sexual orientation questioning among heterosexual men. Men and Masculinities, 12(4), 425-443. https://doi.org/10.1177/1097184X08322630

Nebot-Garcia, J. E., García-Barba, M., Gil-Juliá, B., Giménez-García, C. y Ballester-Arnal, R. (2018). Comportamientos homosexuales en jóvenes heterosexuales: diferencias de género. Revista Àgora de Salut, 5, 59-68. http://dx.doi.org/10.6035/AgoraSalut.2018.5.6

Petersen, J. L. y Hyde, J. S. (2011). Gender differences in sexual attitudes and behaviors: A review of meta-analytic results and large datasets. Journal of Sex Research, 48(2-3), 149-165. https://doi.org/10.1080/00224 499.2011.551851

Poteat, V. P. y Anderson, C. J. (2012). Developmental changes in sexual prejudice from early to late adolescence: the effects of gender, race, and ideology on different patterns of change. Developmental Psychology, 48(5), 1403-1415. https://doi.org/10.1037/a0026906

Shearer, A., Herres, J., Kodish, T., Squitieri, H., James, K., Russon, J.,...Diamond, G. S. (2016). Differences in mental health symptoms across lesbian, gay, bisexual, and questioning youth in primary care settings. Journal of Adolescent Health, 59(1), 38-43. https://doi.org/10.1016/j.jadohealth.2016.02.005

Soriano, S. (2004). Cómo se vive la homosexualidad y el lesbianismo (2ª edición). Salamanca: Amarú Ediciones.

Sutton, G. W. (2016). A house divided: Sexuality, morality, and Christian cultures. Eugene, OR: Pickwick

Vrangalova, Z. y Savin-Williams, R. C. (2012). Mostly heterosexual and mostly gay/lesbian: Evidence for new sexual orientation identities. Archives of Sexual Behavior, 47(1), 85-101. https://doi.org/10.1007/s10508-0129927-y

Yost, M. R. y McCarthy, L. (2012). Girls gone wild? Heterosexual women's same-sex encounters at college parties. Psychology of Women Quarterly, 36(1), 7-24. https://doi.org/10.1177/0361684311414818 